\title{
On the functional equation $x+f(y+f(x))=y+f(x+f(y))$
}

JÜRG RÄTZ

Abstract. For an abelian group $(G,+, 0)$ we consider the functional equation

$$
f: G \rightarrow G, x+f(y+f(x))=y+f(x+f(y)) \quad(\forall x, y \in G),
$$

most times together with the condition

$$
f(0)=0 .
$$

Our main question is whether a solution of $(1) \wedge(0)$ must be additive, i.e., an endomorphism of $G$. We shall answer this question in the negative (Example 3.14) Rätz (Aequationes Math $81: 300,2011)$.

Mathematics Subject Classification (2010). 39B12, 39B52.

Keywords. Abelian groups, composite functional equations.

\section{Introduction, notation, preliminaries, and some history}

We denote by $S(G)$ the set of all solutions of (1) and put

$$
S_{0}(G):=\{f \in S(G) ; f(0)=0\} .
$$

The symbol $:=$ means that the right-hand side defines the left-hand side. $=(\ldots)=$ is a short form of quotation of $(\ldots)$, and $\square$ marks the end of a proof. $i_{A}$ denotes the identity mapping of the set $A$ and $\underline{a}$ the constant mapping with value $a . \mathbb{P}, \mathbb{N}, \mathbb{N}^{0}, \mathbb{Z}, \mathbb{Q}, \mathbb{R}$ stand for the sets of prime numbers, positive integers, nonnegative integers, integers, rational and real numbers, respectively. For every $n \in \mathbb{N}, f^{n}$ means the $n$-th iterate of $f: G \rightarrow G$. Throughout the paper, $(G,+, 0)$ or $(G,+)$ or $G$ denotes an abelian group.

For every $n \in \mathbb{Z}, \omega_{n}: G \rightarrow G$ defined by $\omega_{n}(x):=n x(\forall x \in G)$ is in $\operatorname{End}(G)$, i.e. $\omega_{n}$ is an endomorphism of $G$. For every $z \in G,\langle z\rangle$ denotes the subgroup of $G$ generated by $z$, and $t_{z}: G \rightarrow G$ is the translation $x \mapsto$ $x+z(\forall x \in G)$ of $G$ by $z$. We use 0 for the identity element of $G$ as well as for 
the integer 0 ; it will always be clear from the context what is meant. We freely use the fact that for abelian groups $A$ and $B$ we have $A \times B \cong A \oplus B$, e.g., by denoting the elements of $A \oplus B$ as ordered pairs $(a, b) \in A \times B$ whenever we find it convenient. For every $n \in \mathbb{N}$, we let $\mathbb{Z}_{n}$ stand for the cyclic group with $n$ elements, most times written as $\{0, \ldots, n-1\}$.

The following remarks are easily verified.

Remark 1.1. If $G$ and $H$ are abelian groups and $\varphi: G \rightarrow H$ is an isomorphism, if $f: G \rightarrow G, g: H \rightarrow H, g=\varphi \circ f \circ \varphi^{-1}$, then

(a) $f \in S(G) \Longrightarrow g \in S(H) ; f \in S_{0}(G) \Longrightarrow g \in S_{0}(H)$.

(b) $f \in \operatorname{End}(G) \Longrightarrow g \in \operatorname{End}(H)$.

(c) $S_{0}(G) \subset \operatorname{End}(G) \Longrightarrow S_{0}(H) \subset \operatorname{End}(H)$.

Remark 1.2. The abelian group $G$ is a unitary $\mathbb{Z}$-module in a natural way. We shall tacitly use the corresponding computation rules many times.

In particular, for $f: G \rightarrow G: \quad f(0)=0 \Longleftrightarrow f(n \cdot 0)=n f(0)(\forall n \in \mathbb{Z})$.

In [2], Brillouët-Belluot asked what the continuous functions in $S(\mathbb{R})$ are. By a connectedness argument, Jarczyk and Jarczyk [3] showed that there are none.

Balcerowski [1] found many interesting and fundamental properties of solutions of (1); we list here some of them:

(B1) $f \in S(G) \Longrightarrow f$ is injective.

(B2) $f \in S(G) \Longrightarrow 0 \in f(G)$.

(B3) $f \in S_{0}(G) \Longrightarrow$

$$
f^{2}(x)+x=f(x)(\forall x \in G) .
$$

(B4) $f \in S_{0}(G) \Longrightarrow f^{3}=-i_{G}$ and $f$ is odd.

(B5) $f \in \operatorname{End}(G) \Longrightarrow\left[f \in S_{0}(G) \Longleftrightarrow(3)\right]$.

(B6) $f \in S(G), z \in G \Longrightarrow f \circ t_{z} \in S(G)$.

(B7) $f \in S_{0}(G) \Longrightarrow 2 f \in \operatorname{End}(G)$.

(B8) $f \in S_{0}(G), \omega_{2}$ injective $\Longrightarrow f \in \operatorname{End}(G)$.

(B9) $f \in S(\mathbb{R}) \Longrightarrow f$ is nowhere continuous.

(Cf. [1, Lemma 1, Corollary 1, Remark 1, Corollary 2, Lemma 2, Remark 2, Theorem 1, Corollary 3, Corollary 4]). (B9) strengthens and confirms the main result of [3].

(B10) Open question [1, Remark 3]): Is $S_{0}(G) \subset \operatorname{End}(G)$ true in general? I.e., can the injectivity of $\omega_{2}$ in (B8) be deleted?

(B6) and (B1) above can slightly be sharpened:

Remark 1.3. $\left(\mathrm{B} 6^{\prime}\right) S(G)=\left\{f \circ t_{z} ; f \in S_{0}(G), z \in G\right\}$,

$\left(\mathrm{B}^{\prime}\right) f \in S(G) \Longrightarrow f$ is bijective.

Proof. (B6 $\left.{ }^{\prime}\right): B:=\left\{f \circ t_{z} ; f \in S_{0}(G), z \in G\right\}$. Now $B \subset S(G)$ follows from $S_{0}(G) \subset S(G)$ and (B6). Conversely, let $h \in S(G)$. By (B2) there exists 
$z \in G$ with $h(z)=0$. Define $f:=h \circ t_{z}$, and by (B6) $f \in S(G)$, moreover $f(0)=h(z)=0$, so that we even have $f \in S_{0}(G)$ and $h=f \circ t_{-z}$, so $h \in B$, finally $S(G) \subset B$, in the total $S(G)=B$. - (B1'): If $f \in S(G)$, then by (B6 $\left.{ }^{\prime}\right)$ there are $f^{\prime} \in S_{0}(G)$ and $z \in G$ such that $f=f^{\prime} \circ t_{z}$. By (B4) $\left(f^{\prime}\right)^{3}=-i_{G}$, so $f^{\prime}$ must be bijective, and together with the bijectivity of $t_{z}$ we get that of $f$.

$\left(\mathrm{B} 6^{\prime}\right)$ says that it is sufficient to consider $S_{0}(G)$ rather than $S(G)$ and ensures

$$
S(G) \neq \emptyset \Longleftrightarrow S_{0}(G) \neq \emptyset \text {. }
$$

\section{New results}

Lemma 2.1. (a) $\omega_{1}=i_{G} \in S_{0}(G) \Longleftrightarrow G=\{0\}$.

(b) $\omega_{-1}=-i_{G} \in S_{0}(G) \Longleftrightarrow 3 G=\{0\}$.

Proof. (a) $\Longrightarrow$ : Let $x, y \in G$ be arbitrary. Then $x+y+x={ }_{(1)}=y+x+y$, i.e., $x=y$, so card $G=1, G=\{0\} .-\Longleftarrow$ is trivial.

(b) $\omega_{-1}$ is an involution and in $\operatorname{End}(G)$. So $\omega_{-1} \in S_{0}(G) \Leftarrow{ }_{(B 5)} \Rightarrow \omega_{-1}^{2}+\omega_{1}=$ $\omega_{-1} \Longleftrightarrow 3 \omega_{1}=0 \Longleftrightarrow 3 G=\{0\}$.

Corollary 2.2. There are no continuous functions in $S(\mathbb{R})$.

After the proofs given in [3] and [1] (cf. (B9)), we proceed completely differently.

Proof. Assume $f: \mathbb{R} \rightarrow \mathbb{R}$ is continuous, $f \in S(\mathbb{R})$. By (B2) there exists $z \in \mathbb{R}$ with $f(z)=0$. For $h:=f \circ t_{z}$ we get $h \in S(\mathbb{R})$ by $($ B6) and $h(0)=f(z)=0$, so $h \in S_{0}(\mathbb{R})$. (B4) implies $h^{3}=-i_{\mathbb{R}}$, so $h^{6}=i_{\mathbb{R}}$, moreover $h$ is continuous. By a theorem of McShane [4], $h$ must be an involution in all possible cases, i.e., $h^{2}=i_{\mathbb{R}}$. Together with $h^{3}=-i_{\mathbb{R}}$ we obtain $h=-i_{\mathbb{R}}$, so by Lemma $2.1(\mathrm{~b})$ $h \notin S_{0}(\mathbb{R})$, a contradiction.

Lemma 2.3. Let $\left(G_{i}\right)_{i \in I}$ be a family of abelian groups, $G:=\prod_{i \in I} G_{i}$ their (cartesian) product, $G^{\prime}:=\bigoplus_{i \in I} G_{i}$ their direct sum, $f_{i} \in S_{0}\left(G_{i}\right)(\forall i \in I)$, and $f: G \rightarrow G, f:\left(x_{i}\right)_{i \in I} \mapsto\left(f_{i}\left(x_{i}\right)\right)_{i \in I}$ for all $\left(x_{i}\right)_{i \in I} \in G$. Then:

(a) $f \in S_{0}(G)$.

(b) $f\left(G^{\prime}\right) \subset G^{\prime}$.

(c) If $f^{\prime}: G^{\prime} \rightarrow G^{\prime}$ is the restriction of $f$, then $f^{\prime} \in S_{0}\left(G^{\prime}\right)$.

Proof. (a) is established by a straightforward computation. (b) If $x:=$ $\left(x_{i}\right)_{i \in I} \in G^{\prime}$, then the support of $x$ is finite. Since $f_{i}(0)={ }_{(0)}=0(\forall i \in I)$, the support of $\left(f_{i}\left(x_{i}\right)\right)_{i \in I}$ is finite as well, so $f(x) \in G^{\prime}$. - (c) By (b), the restriction $f^{\prime}: G^{\prime} \rightarrow G^{\prime}$ of $f$ exists. $G^{\prime}$ is a subgroup of $G$, and by (a), $f^{\prime}$ clearly satisfies (1) on $G^{\prime}$ and $(0)$, so $f^{\prime} \in S_{0}\left(G^{\prime}\right)$. 
Lemma 2.4. Every $f \in S(G)$ has exactly one fixed element.

Proof. Let $f \in S(G)$. 1) Let $z, w \in G, f(z)=z, f(w)=w$. Put $x=z, y=w$ in (1): $z+f(w+z)=w+f(z+w)$, so $z=w$, i.e., $f$ has at most one fixed element. - 2) Assume that $f$ has no fixed element. By (B2) there exists $z \in G$ with $f(z)=0$. Put $x=z$ in $(1)$ :

$$
z+f(y+0)=y+f(z+f(y)) \quad(\forall y \in G) .
$$

By $\left(\mathrm{B}^{\prime}\right) f$ is bijective. Define $g: G \rightarrow G, g(y):=f(z+f(y))(\forall y \in G)$, i.e., $g=f \circ t_{z} \circ f$, so

$$
g \text { is bijective. }
$$

By assumption, $f(y) \neq y(\forall y \in G)$, so by $(5) z \neq f(z+f(y))(\forall y \in G)$, i.e. $z \neq g(y)(\forall y \in G)$, in contradiction to (6). Therefore, $f$ has to have at least one fixed element.

Lemma 2.4 confirms and explains Lemma 2.1(a).

Theorem 2.5. If $f \in S_{0}(G)$, then

$$
f(n y)=n f(y) \quad(\forall y \in G, \forall n \in \mathbb{Z}),
$$

i.e., $f$ is $\mathbb{Z}$-homogeneous.

Proof. Let $f \in S_{0}(G)$. By (B1') and (B4), $f$ is bijective and odd. (7) trivially holds for all $y \in G$ and $n=1$ as well as for $n=0$, the latter by (2) or (0). Induction hypothesis: For some $n \in \mathbb{N}$

$$
f(k y)=k f(y) \quad(\forall y \in G, 0 \leq k \leq n)
$$

is assumed to hold.

(1) We first prove three auxiliary assertions:

$$
\begin{gathered}
f(k y)=k f(y) \quad(\forall y \in G,-n \leq k \leq n), \\
f^{-1}(k z)=k f^{-1}(z) \quad(\forall z \in G,-n \leq k \leq n), \\
f\left(y+(n-1) f^{-1}(y)\right)=f(y)+(n-1) y \quad(\forall y \in G) .
\end{gathered}
$$

Proof of $\left({ }^{*}\right)$. Let $y \in G$ be arbitrary. If $0 \leq k \leq n$, then the assertion holds by $(\mathrm{H})$. Let $-n \leq k<0$, so $0<-k \leq n$. By $(\mathrm{H})$ we have $f((-k) y)=(-k) f(y)$. Since $(-k) z=-k z$ (remember Remark 1.2) and $f$ is odd, we get $-f(k y)=-k f(y)$, i.e., $f(k y)=k f(y)$. As $y \in G$ was arbitrary, $(*)$ holds.

Proof of (**). Let $z \in G$ be arbitrary, $-n \leq k \leq n$, and $y:=f^{-1}(z)$. Then $f(k y)={ }_{(*)}=k f(y)$, so $k y=f^{-1} f(k y)=f^{-1}(k f(y))$, i.e., $k f^{-1}(z)=$ $f^{-1}(k f(y))=f^{-1}(k z)$. Since $z \in G$ was arbitrary, $(* *)$ is established.

Proof of $(* * *)$. Let $y \in G$ be arbitrary and $x:=-f((n-1) y)$. Then $(1)$ becomes $-f((n-1) y)+f(y+f(-f((n-1) y)))=y+f(-f((n-1) y)+f(y)) \cdot n \in \mathbb{N}$ 
implies $0 \leq n-1<n$, so by $(\mathrm{H})-(n-1) f(y)+f(y+f(-(n-1) f(y)))=$ $y+f(-(n-1) f(y)+f(y))$ and again by $(\mathrm{H})-(n-1) f(y)+f(y+(n-$ 1) $f(-f(y)))=y+f((-n+2) f(y))$. Now $-n<-n+2 \leq-1+2=1 \leq n$, so $(*)$ yields

$$
-(n-1) f(y)+f(y+(n-1) f(-f(y)))=y+(-n+2) f^{2}(y) .
$$

By (B4), $f$ is odd, so

$$
-(n-1) f(y)+f\left(y-(n-1) f^{2}(y)\right)=y+(-n+2) f^{2}(y) .
$$

Furthermore $-f^{2}(y)=-f^{3} f^{-1}(y)={ }_{(B 4)}=f^{-1}(y)$, therefore

$$
\begin{aligned}
& -(n-1) f(y)+f\left(y+(n-1) f^{-1}(y)\right)=y+(n-2) f^{-1}(y), \text { i.e., } \\
& f\left(y+(n-1) f^{-1}(y)\right)=y+(n-1) f(y)+(n-2) f^{-1}(y) .
\end{aligned}
$$

The right-hand side of $(8)$ is $y+f(y)+(n-2)\left(f(y)+f^{-1}(y)\right)=y+f(y)+(n-$ 2) $\left(f^{2} f^{-1}(y)+f^{-1}(y)\right)={ }_{(3)}=y+f(y)+(n-2) f f^{-1}(y)=y+f(y)+(n-2) y=$ $f(y)+(n-1) y$, so by $(8) f\left(y+(n-1) f^{-1}(y)\right)=f(y)+(n-1) y$. Since $y \in G$ was arbitrary, $(* * *)$ holds.

(2) For arbitrary $y \in G$ and $x:=f^{-1}(n y)$, (1) becomes $f^{-1}(n y)+$ $f\left(y+f f^{-1}(n y)\right)=y+f\left(f^{-1}(n y)+f(y)\right)$, i.e., $f^{-1}(n y)+f((n+1) y)=$ $y+f\left(f^{-1}(n y)+f(y)\right)$, so by $(* *)$

$$
n f^{-1}(y)+f((n+1) y)=y+f\left(n f^{-1}(y)+f(y)\right) .
$$

The right-hand side of (9) is $y+f\left((n-1) f^{-1}(y)+f^{-1}(y)+f(y)\right)={ }_{(3)}=$ $y+f\left((n-1) f^{-1}(y)+y\right)=_{(* * *)}=y+f(y)+(n-1) y=f(y)+n y$, so (9) is $n f^{-1}(y)+f((n+1) y)=f(y)+n y$, i.e.,

$$
f((n+1) y)=f(y)+n y-n f^{-1}(y) .
$$

The right-hand side of (10) is $f(y)+n\left(y-f^{-1}(y)\right)=f(y)+n\left(f f^{-1}(y)-\right.$ $\left.f^{-1}(y)\right)={ }_{(3)}=f(y)+n f^{2} f^{-1}(y)=f(y)+n f(y)=(n+1) f(y)$, so (10) is $f((n+1) y)=(n+1) f(y)$. As $y \in G$ was arbitrary, we have $f((n+1) y)=$ $(n+1) f(y)(\forall y \in G)$. By the so-called second principle of induction, we have so far

$$
f(n y)=n f(y) \quad\left(\forall y \in G, \forall n \in \mathbb{N}^{0}\right) .
$$

(3) Let $y \in G$ be arbitrary, $n \in \mathbb{Z}, n<0$, hence $(-n) \in \mathbb{N}^{0}$. Then $f(n y)=$ $f((-n)(-y))=_{(11)}=(-n) f(-y)={ }_{(B 4)}=(-n)(-f(y))=n f(y)$. Together with (11), we have reached (7).

Corollary 2.6. If $G$ is cyclic and $f \in S_{0}(G)$, then $f \in \operatorname{End}(G)$, i.e., $f$ is additive.

Proof. Say $G=\langle a\rangle$ for some $a \in G$. Let $x, y \in G$ be arbitrary. Then $x=$ $k a, y=\ell a$ for suitable $k, \ell \in \mathbb{Z}$. So $f(x+y)=f(k a+\ell a)=f((k+\ell) a)={ }_{(7)}=$ $(k+\ell) f(a)=k f(a)+\ell f(a)={ }_{(7)}=f(k a)+f(\ell a)=f(x)+f(y)$. Since $x, y \in G$ were arbitrary, $f \in \operatorname{End}(G)$. 
Example 2.7. $S(\mathbb{Z})=\emptyset$.

In fact: assume $f \in S_{0}(\mathbb{Z})$. By Corollary $2.6 f \in \operatorname{End}(\mathbb{Z})$, so by $\left(\mathrm{B1}^{\prime}\right) f \in$ $\operatorname{Aut}(\mathbb{Z})=\left\{i_{\mathbb{Z}},-i_{\mathbb{Z}}\right\}$. By Lemma $2.1 f \notin S_{0}(\mathbb{Z})$, contradicting the assumption. So $S_{0}(\mathbb{Z})=\emptyset$, and by $(4) S(\mathbb{Z})=\emptyset$.

Remark 2.8. The integers

$$
m_{k}:=k^{2}-k+1 \quad(\forall k \in \mathbb{Z})
$$

are positive and odd and satisfy

$$
m_{-k}=m_{k+1} \quad(\forall k \in \mathbb{Z}) .
$$

Lemma 2.9. $k \in \mathbb{Z} \Longrightarrow\left[\omega_{k} \in S_{0}(G) \Longleftrightarrow m_{k} G=\{0\}\right]$.

Proof. Let $k \in \mathbb{Z}$ be arbitrary. Since $\omega_{k} \in \operatorname{End}(G)$, we get from (B5) $\omega_{k} \in$ $S_{0}(G) \Longleftrightarrow \omega_{k}^{2}+\omega_{1}=\omega_{k} \Longleftrightarrow \omega_{k^{2}-k+1}=\underline{0} \Longleftrightarrow m_{k} G=\{0\}$.

Remark 2.10. For every $n \in \mathbb{N}$ we have $\operatorname{End}\left(\mathbb{Z}_{n}\right)=\left\{\omega_{k}: \mathbb{Z}_{n} \rightarrow \mathbb{Z}_{n} ; k \in \mathbb{Z}\right\}$. But here $\omega_{n+k}=\omega_{k}(\forall k \in \mathbb{Z})$, so $\operatorname{End}\left(\mathbb{Z}_{n}\right)=\left\{\omega_{0}, \ldots, \omega_{n-1}\right\}$. Since $m_{k} \mathbb{Z}_{n}=$ $\{0\} \Longleftrightarrow n \mid m_{k}(\forall k \in \mathbb{Z}, \forall n \in \mathbb{N})$, we obtain from Lemma 2.9

$$
S_{0}\left(\mathbb{Z}_{n}\right)=\left\{\omega_{k} ; k \in\{0, \ldots, n-1\}, n \mid m_{k}\right\} \quad(\forall n \in \mathbb{N}) .
$$

Corollary 2.11. If $n \in \mathbb{N}$ is even, then $S\left(\mathbb{Z}_{n}\right)=\emptyset$.

Proof. Since $n$ is even and $m_{k}$ odd $(\forall k \in \mathbb{Z}),(14)$ implies $S_{0}\left(\mathbb{Z}_{n}\right)=\emptyset$, so by (4) $S\left(\mathbb{Z}_{n}\right)=\emptyset$.

Example 2.12. It follows from (14) that $S_{0}\left(\mathbb{Z}_{1}\right)=\left\{\omega_{0}\right\}$ [cf. Lemma 2.1(a)], $S_{0}\left(\mathbb{Z}_{3}\right)=\left\{\omega_{2}\right\}$ [cf. Lemma 2.1(b)], $S_{0}\left(\mathbb{Z}_{5}\right)=\emptyset, S_{0}\left(\mathbb{Z}_{7}\right)=\left\{\omega_{3}, \omega_{5}\right\}, S_{0}\left(\mathbb{Z}_{9}\right)=$ $\emptyset, S_{0}\left(\mathbb{Z}_{11}\right)=\emptyset$.

Example 2.13. $S(\mathbb{Q})=\emptyset$.

In fact: Let $f \in S_{0}(\mathbb{Q})$. By $(\mathrm{B} 8), f \in \operatorname{End}(\mathbb{Q},+)$, so by $\left(\mathrm{B}^{\prime}\right) f \in \operatorname{Aut}(\mathbb{Q},+)$. So there exists $c \in \mathbb{Q} \backslash\{0\}$ with $f(x)=c x(\forall x \in \mathbb{Q})$. From (B3) we get $c^{2} x+x=c x(\forall x \in \mathbb{Q}$ ), therefore (put $x=1) c^{2}+1=c$. But $c^{2}-c+1=$ $\left(c-\frac{1}{2}\right)^{2}-\frac{1}{4}+1>0(\forall c \in \mathbb{Q})$, so $c$ cannot exist. This means $S_{0}(\mathbb{Q})=\emptyset$, and by $(4) S(\mathbb{Q})=\emptyset$.

Remark 2.14. For $p \in \mathbb{P}$ and $m \in \mathbb{N}, \mathbb{Z}_{p}^{m}$ is the additive group of the Galois field $\operatorname{GF}\left(p^{m}\right)$; if moreover $p$ is odd, then $\omega_{2}: \mathbb{Z}_{p}^{m} \rightarrow \mathbb{Z}_{p}^{m}$ is bijective, so by (B8) $S_{0}\left(\mathbb{Z}_{p}^{m}\right) \subset \operatorname{End}\left(\mathbb{Z}_{p}^{m}\right)$. Furthermore by $\left(\mathrm{B} 1^{\prime}\right)$ and (B5)

$$
S_{0}\left(\mathbb{Z}_{p}^{m}\right)=\left\{f \in \operatorname{Aut}\left(\mathbb{Z}_{p}^{m}\right) ; f \text { satisfies }(3)\right\} \quad(p \in \mathbb{P} \text { odd, } m \in \mathbb{N}) .
$$

We shall see in the next section that the situation for $p=2$ is quite different. 


\section{The case $2 G=\{0\}$}

The condition $2 G=\{0\}$ means that $G$ is an elementary abelian 2-group, so (isomorphic to) a $\mathbb{Z}_{2}$-vector space [cf., e.g., [6], p.82, (9.14)]. In this section, where $2 G=\{0\}, \operatorname{dim} G$ will always stand for $\operatorname{dim}_{\mathbb{Z}_{2}} G$.

Remark 3.1. For $2 G=\{0\}$ and every $f: G \rightarrow G$ with $f(0)=0$, we have

(a) $f$ is $\mathbb{Z}$-homogeneous.

(b) $f$ is even and odd.

(c) $2 f=\underline{0} \in \operatorname{End}(G)$.

In fact: (a), (b), (c) follow from

$$
n y=\left\{\begin{array}{ll}
0 & n \in 2 \mathbb{Z} \\
y & n \in 2 \mathbb{Z}+1
\end{array} \quad(\forall y \in G) .\right.
$$

Therefore Theorem 2.5, the second half of (B4) as well as (B7) lose their power in the process of finding $S_{0}(G)$ in the case $2 G=\{0\}$.

Lemma 3.2. If $2 G=\{0\}$ and $f \in S_{0}(G)$, then $G$ is the disjoint union of $C_{0}:=\{0\}$ and, for $G \neq\{0\}$, of 3-cycles $C_{x}:=\{x, f(x), x+f(x)\}(\forall x \in G \backslash\{0\})$ of $f$.

Proof. Let $f \in S_{0}(G)$ be arbitrary. By $\left(\mathrm{B} 1^{\prime}\right), f$ is bijective. Define

$$
x, y \in G ; x \sim_{f} y: \Longleftrightarrow \exists k \in \mathbb{Z} \text { with } y=f^{k}(x) .
$$

Then $\sim_{f}$ is an equivalence relation on $G$; let $C_{x}$ denote the $\sim_{f}$-class of $x(\forall x \in G)$. By (B4) $f^{3}=-i_{G}={ }_{(16)}=i_{G}$. So $C_{x}=\left\{x, f(x), f^{2}(x)\right\}={ }_{(B 3)}=$ $\{x, f(x),-x+f(x)\}={ }_{(16)}=\{x, f(x), x+f(x)\}(\forall x \in G)$, and this automatically becomes $\{0\}$ for $x=0$, while for $x \in G \backslash\{0\},\left(B 1^{\prime}\right),(0)$, and Lemma 2.4 ensure card $C_{x}=3$.

Lemma 3.3. If $2 G=\{0\}, f \in S_{0}(G)$, and

$$
H_{x}:=C_{x} \cup\{0\} \quad(\forall x \in G),
$$

then $H_{0}=\{0\}, H_{x} \cong \mathbb{Z}_{2}^{2}(\forall x \in G \backslash\{0\})$, and

$$
f\left(H_{x}\right)=H_{x} \quad(\forall x \in G) .
$$

So all $H_{x}(x \in G)$ are $f$-invariant subgroups (subspaces) of $G$, and if $x \neq 0, H_{x}$ is isomorphic to the Klein four group.

Proof. (B3) and (16) imply $c=a+b$ for all pairwise distinct $a, b, c \in C_{x}$ for $x \neq 0$. Moreover $2 a=2 b=2 c=0$, so $H_{x} \cong \mathbb{Z}_{2}^{2}(\forall x \in G \backslash\{0\})$ is clear, and $H_{0}=\{0\}$ is trivial. As cycles of $f$, the $C_{x}(x \in G)$ satisfy $f\left(C_{x}\right)=C_{x}$, and (0) and (17) imply (18).

Example 3.4. Let $2 G=\{0\}$. Then

(a) $\operatorname{dim} G=0 \Longrightarrow S_{0}(G)=\left\{i_{G}\right\}$. 
(b) $\operatorname{dim} G=1 \Longrightarrow S_{0}(G)=\emptyset$.

(c) $\operatorname{dim} G=2 \Longrightarrow S_{0}(G)=\left\{f_{1}, f_{2}\right\}$, where $f_{1}, f_{2}$ are the two permutations of $G$ with 0 as their unique fixed element, and these are additive.

Proof. (a) follows from Lemma 2.1(a). (b) We have $G \cong \mathbb{Z}_{2}$. By Corollary 2.11, $S_{0}\left(\mathbb{Z}_{2}\right)=\emptyset$, so by Remark $1.1, S_{0}(G)=\emptyset$. (c) Let $f \in S_{0}(G)$. By Lemma 3.2 , $f$ must have the 1-cycle $C_{0}=\{0\}$ and a unique 3 -cycle, say $\{a, b, c\}$, disjoint to $\{0\}$. So, in cycle notation, $f=(0)(a b c)=: f_{1}$ or $f=(0)(a c b)=: f_{2}$, so $S_{0}(G) \subset\left\{f_{1}, f_{2}\right\}$. Moreover,

$$
f_{1}^{2}=f_{2} \quad \text { and } \quad f_{2}^{2}=f_{1} .
$$

Conversely, let $f \in\left\{f_{1}, f_{2}\right\}$ and $x, y \in G$ be arbitrary.

Case 1: $x=y$. Then $f_{1}(x+y)=f_{1}(x+x)=f_{1}(0)=0=f_{1}(x)+f_{1}(x)=$ $f_{1}(x)+f_{1}(y)$.

Case 2: $x \neq y$.

Case 2a: $x=0, y \neq 0$. Then $f_{1}(x+y)=f_{1}(y)=0+f_{1}(y)=f_{1}(0)+f_{1}(y)=$ $f_{1}(x)+f_{1}(y)$.

Case 2b: $x \neq 0, y \neq 0$, say $x=a, y=b$.

Then $f_{1}(x+y)=f_{1}(a+b)=$ Lemma $3.3=f_{1}(c)=a=_{\text {Lemma } 3.3}=b+c=$ $f_{1}(a)+f_{1}(b)=f_{1}(x)+f_{1}(y)$.

Thus in all three cases $f_{1}(x+y)=f_{1}(x)+f_{1}(y)$. Since $x, y \in G$ were arbitrary, $f_{1} \in \operatorname{End}(G)$, hence $f_{2}={ }_{(19)}=f_{1}^{2} \in \operatorname{End}(G)$. Furthermore,

$$
f_{1}+f_{2}=\frac{0 a b \quad b \quad c}{0 b+c c+a a+b}=\frac{0 a b c}{0 a b c}=i_{G}
$$

hence $f_{1}^{2}+i_{G}={ }_{(19)}=f_{2}+i_{G}={ }_{(20)}=f_{2}+\left(f_{1}+f_{2}\right)=f_{1}$ and analogously $f_{2}^{2}+i_{G}=f_{2}$. Therefore, $f_{1}$ and $f_{2}$ satisfy (3), and by (B5) $f_{1}, f_{2} \in S_{0}(G)$. In total, $S_{0}(G)=\left\{f_{1}, f_{2}\right\}$.

Corollary 3.5. $2 G=\{0\}, f \in S_{0}(G), x \in G \Longrightarrow f$ is additive on $H_{x}$.

Proof. By (18) $f\left(H_{x}\right)=H_{x}$ and by Lemma $3.3 \operatorname{dim} H_{x} \in\{0,2\}$. Now the assertion follows from Example 3.4(a) and (c).

Remark 3.6. For $G \cong \mathbb{Z}_{2}^{2}$ we got $S_{0}(G) \subset \operatorname{End}(G)$ in Example 3.4(c), in the absence of injectivity of $\omega_{2}: G \rightarrow G$. So this latter condition is sufficient for $S_{0}(G) \subset \operatorname{End}(G)$ by (B8), but by no means necessary, as already noted in $[1$, p. 300, Remark 3].

Lemma 3.7. Let $2 G=\{0\}$. Then

(a) If $n \in \mathbb{N}^{0}, \operatorname{dim} G=n$, then $\left[S_{0}(G) \neq \emptyset \Longleftrightarrow n\right.$ is even $]$.

(b) If $\operatorname{dim} G \geq \aleph_{0}$, then $S_{0}(G) \neq \emptyset$. 
Proof. (a) Clearly $\left.G \cong \mathbb{Z}_{2}^{n} .1\right) \Longrightarrow$ : Let $S_{0}(G) \neq \emptyset, f \in S_{0}(G)$. By Lemma 3.2

$$
2^{n}=\operatorname{card} G \equiv_{3} 1 \text {. }
$$

Assume that $n=2 k+1\left(\exists k \in \mathbb{N}^{0}\right)$. Then $2^{n}=2^{2 k+1}=4^{k} \cdot 2 \equiv{ }_{3} 1 \cdot 2=2$, a contradiction to (21). So $n$ must be even. 2$) \Longleftarrow$ : For $n=0, S_{0}(G) \neq \emptyset$ by Example 3.4(a). Let $n \in \mathbb{N}$ be even. Then $G=\mathbb{Z}_{2}^{2} \oplus \ldots \oplus \mathbb{Z}_{2}^{2}(n / 2$ direct summands) by the associativity of $\oplus$. By Example 3.4(c) $S_{0}\left(\mathbb{Z}_{2}^{2}\right) \neq \emptyset$, so by Lemma $2.3, S_{0}(G) \neq \emptyset$.

(b) There exists an infinite set $J$ with $G \cong\left(\mathbb{Z}_{2}\right)^{(J)}$ (direct sum of card $J$ copies of $\left.\mathbb{Z}_{2}\right)$. If we put $J_{0}:=J \times\{0\}, J_{1}:=J \times\{1\}$, then $\operatorname{card}\left(J_{0} \cup J_{1}\right)=$ card $J$, i.e. $G \cong\left(\mathbb{Z}_{2}\right)^{\left(J_{0} \cup J_{1}\right)}$. Now the sets $I_{j}:=\{(j, 0),(j, 1)\}(j \in J)$ form a partition of $J_{0} \cup J_{1}$, so $\bigoplus_{j \in J}\left(\mathbb{Z}_{2}\right)^{I_{j}} \cong \mathbb{Z}_{2}^{\left(J_{0} \cup J_{1}\right)} \cong G$. Since card $I_{j}=$ $2(\forall j \in J)$, we have $\left(\mathbb{Z}_{2}\right)^{I_{j}} \cong \mathbb{Z}_{2}^{2}(\forall j \in J)$, so $G \cong\left(\mathbb{Z}_{2}^{2}\right)^{(J)}$. By Example 3.4(c) $S_{0}\left(\mathbb{Z}_{2}^{2}\right) \neq \emptyset$, so by Lemma 2.3(c) and Remark 1.1(a) $S_{0}(G) \neq \emptyset$.

Remark 3.8. Lemma 3.7 implies that $S_{0}\left(\mathbb{Z}_{2}^{n}\right)=\emptyset$ for odd $n \in \mathbb{N}$ and $S_{0}\left(\mathbb{Z}_{2}^{4}\right) \neq \emptyset$. For $n \in \mathbb{N}^{0}, n \leq 3$, we have seen so far (cf. Example 3.4) that $S_{0}\left(\mathbb{Z}_{2}^{n}\right) \subset \operatorname{End}\left(\mathbb{Z}_{2}^{n}\right)$. Does this also hold for $n=4$ ?

Theorem 3.9. $S_{0}\left(\mathbb{Z}_{2}^{4}\right) \subset \operatorname{End}\left(\mathbb{Z}_{2}^{4}\right)$.

Proof. Let $f \in S_{0}\left(\mathbb{Z}_{2}^{4}\right)$ as well as $x, y \in \mathbb{Z}_{2}^{4}$ be arbitrary but fixed in the following.

Case 1. $H_{x} \subset H_{y}$ and/or $H_{y} \subset H_{x}$. Then $H_{x} \cup H_{y}$ is the larger one of $H_{x}, H_{y}$. By Corollary 3.5, $f$ is additive on $H_{x} \cup H_{y}$. Since by (17) $x, y \in H_{x} \cup H_{y}$, we have

$$
f(x+y)=f(x)+f(y) .
$$

Case 2. $H_{x} \not \subset H_{y}$ and $H_{y} \not \subset H_{x}$. Then $x \neq 0, y \neq 0$. By (17), $C_{x}=C_{y}$ would imply $H_{x}=H_{y}$, which is excluded in Case 2 . So $C_{x} \neq C_{y}$, hence, as $\sim_{f}$-classes, $C_{x} \cap C_{y}=\emptyset$, and by (17) $H_{x} \cap H_{y}=\{0\}$. By Lemma 3.3, $\operatorname{dim} H_{x}=\operatorname{dim} H_{y}=2$, hence $H_{x} \oplus H_{y}=\mathbb{Z}_{2}^{4}$. It is clear from Lemma 3.2 and card $\mathbb{Z}_{2}^{4}=16$, that $\mathbb{Z}_{2}^{4}$ is the disjoint union of $C_{0}=\{0\}$ and five 3-cycles of $f$.

We first prove three auxiliary statements (3.9.1), (3.9.2), (3.9.3).

(3.9.1). $x+y \neq 0, x+f(y) \neq 0, y+f(x) \neq 0$.

In fact, $x+y=0$ would imply $x=y$, in contradiction to $C_{x} \cap C_{y}=\emptyset$. Furthermore, $x+f(y)=0$ would lead to $f(y)=x$, i.e., to $x \sim_{f} y$, also contradicting $C_{x} \cap C_{y}=\emptyset$, and the third formula is obtained analogously.

(3.9.2). $C_{x}, C_{y}, C_{x+y}, C_{x+f(y)}, C_{y+f(x)}$ are pairwise disjoint.

In fact, $C_{x} \cap C_{y}=\emptyset$ was already an immediate consequence of Case 2 . 
Assume $C_{x} \cap C_{x+y} \neq \emptyset$. As $\sim_{f}$-classes, $C_{x}=C_{x+y}$, we have $x+y \in C_{x} \subset$ $H_{x}$. But also $x \in H_{x}$, so $y=x+y+x \in H_{x}$. Since $y \neq 0$, we have by (17) $y \in C_{x}$, so $y \in C_{x} \cap C_{y}$, a contradiction. Therefore $C_{x} \cap C_{x+y}=\emptyset$ and, analogously, $C_{y} \cap C_{x+y}=\emptyset$.

Assume $x+f(y) \sim_{f} x$. Then $x+f(y) \in C_{x} \subset H_{x}$ and $f(y)=x+x+f(y) \in$ $H_{x}$. Now $y \neq 0,(0)$ and $\left(\mathrm{B}^{\prime}\right)$ require $f(y) \neq 0$, so by $(17) f(y) \in C_{x}$. But also $f(y) \in C_{y}$, a contradiction. So $C_{x+f(y)} \cap C_{x}=\emptyset$ and analogously $C_{y+f(x)} \cap C_{y}=\emptyset$.

Suppose $x \sim_{f} y+f(x)$. Then $y+f(x) \in C_{x} \subset H_{x}$, so $y=y+f(x)+f(x) \in$ $H_{x}$, so, since $y \neq 0$, we have $y \in C_{x}$, i.e., $y \in C_{x} \cap C_{y}$, a contradiction. Therefore $C_{x} \cap C_{y+f(x)}=\emptyset$ and analogously $C_{y} \cap C_{x+f(y)}=\emptyset$.

Assume $x+y \sim_{f} y+f(x)$. Then $y+f(x) \in C_{x+y} \subset H_{x+y}$, so $x+f(x)=$ $x+y+y+f(x) \in H_{x+y}$. Because of (0), $x \neq 0$ and Lemma $2.4 f(x) \neq x$, so $x+f(x) \neq 0$, i.e., $x+f(x) \in C_{x+y}$, so $x+f(x) \in C_{x} \cap C_{x+y}$, which was already recognized above to be impossible. So $C_{x+y} \cap C_{y+f(x)}=\emptyset$ and analogously $C_{x+y} \cap C_{x+f(y)}=\emptyset$.

Finally suppose $x+f(y) \sim_{f} y+f(x)$. Since $x+f(y) \sim_{f} f(x+f(y))$, we have $f(x+f(y)) \in C_{y+f(x)} \subset H_{y+f(x)}$ and furthermore $f(x+f(y))+f(y+f(x)) \in$ $H_{y+f(x)}$, so by (1) (remember $\left.f \in S_{0}\left(\mathbb{Z}_{2}^{4}\right)\right) x+y \in H_{y+f(x)}$. By (3.9.1) $x+y \neq 0$, so $x+y \in C_{y+f(x)}$, in contradiction to $C_{x+y} \cap C_{y+f(x)}=\emptyset$, which is already established. Hence $C_{x+f(y)} \cap C_{y+f(x)}=\emptyset$.

(3.9.3). $f(x)+f(y) \in C_{x+y}$.

In fact, by (3.9.1) and (3.9.2), $C_{x}, C_{y}, C_{x+y}, C_{x+f(y)}, C_{y+f(x)}$ are the five 3 cycles of $f$, hence

$$
\mathbb{Z}_{2}^{4}=C_{0} \dot{\cup} C_{x} \dot{\cup} C_{y} \dot{\cup} C_{x+y} \dot{\cup} C_{x+f(y)} \dot{\cup} C_{y+f(x)}
$$

$f(x)+f(y) \in C_{0}=\{0\}$ would imply $f(x)=f(y)$, so by (B1) $x=y$, in contradiction to Case 2. So $f(x)+f(y) \notin C_{0}$.

Assume $f(x)+f(y) \in C_{x}$. Then $f(x)+f(y) \in H_{x}, f(y)=f(x)+f(x)+$ $f(y) \in H_{x}$, so $f(y) \in H_{x} \cap H_{y}={ }_{\text {Case } 2}=\{0\}$, i.e., $f(y)=0$, so by (B1) and (0) $y=0$, a contradiction. Therefore $f(x)+f(y) \notin C_{x}$, and analogously $f(x)+f(y) \notin C_{y}$.

Suppose $f(x)+f(y) \in C_{x+f(y)} \subset H_{x+f(y)}$. Then $x+f(x)=x+f(y)+$ $f(x)+f(y) \in H_{x+f(y)}$. But $x+f(x) \in_{\text {Lemma } 3.2} \in C_{x}$, so $x+f(x) \neq 0$, i.e., $x+f(x) \in C_{x} \cap C_{x+f(y)}$, a contradiction to (3.9.2). So $f(x)+f(y) \notin C_{x+f(y)}$ and analogously $f(x)+f(y) \notin C_{y+f(x)}$.

Now it follows from (23) that $f(x)+f(y) \in C_{x+y}$, and (3.9.3) is proved. (3.9.3) says by Lemma 3.2 that

$$
f(x)+f(y) \in\{x+y, f(x+y), x+y+f(x+y)\} .
$$


Case 2a. $f(x)+f(y)=x+y$. So $y+f(x)=x+f(y)$, i.e. $f(y+f(x))=$ $f(x+f(y))$, so by (1) $x=y$, which is excluded in Case 2. Therefore, Case 2a is impossible.

Case 2b. $f(x)+f(y)=x+y+f(x+y)$. We replace $y$ by $y+f(x)$ in (1) and obtain $x+f(y+f(x)+f(x))=y+f(x)+f(x+f(y+f(x)))$, i.e. $x+f(y)=$ $y+f(x)+f(x+f(y+f(x)))$, i.e., $x+y+f(x)+f(y)=f(x+f(y+f(x)))$, so by the assumption of Case $2 \mathrm{~b} f(x+y)=f(x+f(y+f(x)))$, and by (B1) $x+y=x+f(y+f(x))$, i.e., $y=f(y+f(x))$. But $f(y+f(x)) \in C_{y+f(x)}$, so $y \in C_{y} \cap C_{y+f(x)}$, in contradiction to (3.9.2). Therefore, Case 2b is impossible too.

From (24) we get (22) $f(x)+f(y)=f(x+y)$. Therefore, (22) holds in the two complementary cases 1 and 2 . Since $x, y \in \mathbb{Z}_{2}^{4}$ were arbitrary, we have $f \in \operatorname{End}\left(\mathbb{Z}_{2}^{4}\right)$, and Theorem 3.9 is proved.

Remark 3.10. In (3.9.2), among other things, $x+y \chi_{f} x+f(y)$ was established. This shows that + and $\sim_{f}$ are not compatible in the sense that $z, w, z^{\prime}, w^{\prime} \in$ $G ; z \sim_{f} z^{\prime}, w \sim_{f} w^{\prime} \not z+w \sim_{f} z^{\prime}+w^{\prime}$. So $\sim_{f}$ is not a congruence relation on the group $(G,+)$.

Corollary 3.11. If $2 G=\{0\}, f \in S_{0}(G)$ and $M$ is a 4-dimensional subspace of $G$ with $f(M)=M$, then $f$ is additive on $M$.

Proof. We have $M \cong \mathbb{Z}_{2}^{4}$, and the restriction $\tilde{f}: M \rightarrow M$ of $f$ is available and in $S_{0}(M)$. By Theorem 3.9 and Remark 1.1(c) $S_{0}(M) \subset \operatorname{End}(M)$, so $\tilde{f} \in \operatorname{End}(M)$, i.e., $f$ is additive on $M$.

Corollary 3.12. If $2 G=\{0\}, f \in S_{0}(G)$, and $f(M)=M$ for all 4-dimensional subspaces of $G$, then $f \in \operatorname{End}(G)$.

Proof. Let $x, y \in G$ be arbitrary. If $H_{x} \subset H_{y}$ and/or $H_{y} \subset H_{x}$, we proceed as in Case 1 of the proof of Theorem 3.9 to get (22) $f(x+y)=f(x)+f(y)$. In the opposite case, $\operatorname{dim} M=4$ for $M:=H_{x} \oplus H_{y}$, and by hypothesis we have $f\left(H_{x} \oplus H_{y}\right)=H_{x} \oplus H_{y}$. By Corollary 3.11, we arrive again at (22). Since $x, y \in G$ were arbitrary, $f \in \operatorname{End}(G)$.

Remark 3.13. Corollaries 3.11 and 3.12 show the way how to possibly obtain a non-additive $f \in S_{0}\left(\mathbb{Z}_{2}^{6}\right)$; remember $S_{0}\left(\mathbb{Z}_{2}^{5}\right)=\emptyset$ by Lemma 3.7(a). The experience gained in the proof of Theorem 3.9 is that $\mathbb{Z}_{2}^{4}$ is just narrow enough for enforcing an $f \in S_{0}\left(\mathbb{Z}_{2}^{4}\right)$ to be additive.

Now we come to a serious contrast to Remark 3.8 and Theorem 3.9.

Example 3.14. $S_{0}\left(\mathbb{Z}_{2}^{6}\right) \not \subset \operatorname{End}\left(\mathbb{Z}_{2}^{6}\right)$, so the open question (B10) in Sect. 1 is answered in the negative. 
Proof. 1) First we do some heuristics for finding a function in $S_{0}\left(\mathbb{Z}_{2}^{6}\right) \backslash \operatorname{End}\left(\mathbb{Z}_{2}^{6}\right)$. We assume that $f$ is such a function. Non-additivity of $f$ is manifested by the existence of two elements of $\mathbb{Z}_{2}^{6}$, say $e_{1}$ and $e_{3}$, with

$$
f\left(e_{1}+e_{3}\right) \neq f\left(e_{1}\right)+f\left(e_{3}\right) .
$$

By Lemmas 3.2 and 3.3, $C_{x}$ and $H_{x}\left(x \in \mathbb{Z}_{2}^{6}\right)$ are available for $f$. It follows from (25) and Corollary 3.5 that $H_{e_{1}} \not \subset H_{e_{3}}$ and $H_{e_{3}} \not \subset H_{e_{1}}$, therefore $e_{1} \neq 0, e_{3} \neq$ $0, C_{e_{1}} \cap C_{e_{3}}=\emptyset, H_{e_{1}} \cap H_{e_{3}}=\{0\}, \operatorname{dim} H_{e_{1}}=\operatorname{dim} H_{e_{3}}=2$ and $\operatorname{dim}\left(H_{e_{1}} \oplus\right.$ $\left.H_{e_{3}}\right)=4$; cf. the beginning of Case 2 in the proof of Theorem 3.9. We now put

$$
e_{2}:=f\left(e_{1}\right) \in H_{e_{1}}, \quad e_{4}:=f\left(e_{3}\right) \in H_{e_{3}}
$$

and see that $\left\{e_{1}, e_{2}, e_{3}, e_{4}\right\}$ is a basis of the subspace $H_{e_{1}} \oplus H_{e_{3}}$. Assume for a moment that $f\left(H_{e_{1}} \oplus H_{e_{3}}\right) \subset H_{e_{1}} \oplus H_{e_{3}}$. Then, since $f$ is injective by (B1) and $H_{e_{1}} \oplus H_{e_{3}}$ is finite, we would have $f\left(H_{e_{1}} \oplus H_{e_{3}}\right)=H_{e_{1}} \oplus H_{e_{3}}$, so by Corollary 3.11, $f$ would be additive on $H_{e_{1}} \oplus H_{e_{3}}$, in contradiction to (25). Therefore

$$
f\left(H_{e_{1}} \oplus H_{e_{3}}\right) \not \subset H_{e_{1}} \oplus H_{e_{3}} .
$$

More explicitely, we find

$$
f\left(e_{1}+e_{3}\right) \notin H_{e_{1}} \oplus H_{e_{3}} .
$$

Namely, (0), (B1), Lemma 2.4, (25), and the pairwise disjointness of $C_{e_{1}}, C_{e_{3}}, C_{e_{1}+e_{3}}, C_{e_{1}+f\left(e_{3}\right)}, C_{e_{3}+f\left(e_{1}\right)}$ (true in analogy to $(3.9 .2)$ ) prevent the equality of $f\left(e_{1}+e_{3}\right)$ to any one of the elements of $H_{e_{1}} \oplus H_{e_{3}}$. Because $H_{e_{2}}={ }_{(26)}=H_{f\left(e_{1}\right)}=H_{e_{1}}$ and $H_{e_{4}}=H_{e_{3}}$, we get similarly

$$
f\left(e_{2}+e_{4}\right) \notin H_{e_{1}} \oplus H_{e_{3}},
$$

and we define

$$
e_{5}:=f\left(e_{1}+e_{3}\right), \quad e_{6}:=f\left(e_{2}+e_{4}\right) .
$$

Furthermore, (B4), the linear independence of $\left\{e_{1}, \ldots, e_{4}\right\},(28),(29)$ lead to $e_{1}+e_{3} \chi_{f} e_{2}+e_{4}$, so to $H_{e_{1}+e_{3}} \cap H_{e_{2}+e_{4}}=\{0\}$. So by (30), $\left\{e_{5}, e_{6}\right\}$ is linearly independent and $e_{5}, e_{6} \notin H_{e_{1}} \oplus H_{e_{3}}$. Therefore, $\left\{e_{1}, e_{2}, e_{3}, e_{4}, e_{5}, e_{6}\right\}$ is a basis of $\mathbb{Z}_{2}^{6}$. By Lemma 3.2 and because of card $\mathbb{Z}_{2}^{6}=64, \mathbb{Z}_{2}^{6}$ is the disjoint union of $C_{0}=\{0\}$ and 213 -cycles of $f$. So far some heuristic thoughts on what an $f \in S_{0}\left(\mathbb{Z}_{2}^{6}\right) \backslash \operatorname{End}\left(\mathbb{Z}_{2}^{6}\right)$ has to look like.

2) On the basis of part 1) of this proof and keeping in mind (3) and special cases of (1) as necessary conditions for a function to belong to $S_{0}\left(\mathbb{Z}_{2}^{6}\right)$, we now construct the bijective function $f_{0}: \mathbb{Z}_{2}^{6} \rightarrow \mathbb{Z}_{2}^{6}$ as follows:

2.1) Let $\left\{e_{1}, e_{2}, e_{3}, e_{4}, e_{5}, e_{6}\right\}$ be a basis of $\mathbb{Z}_{2}^{6}$ and put

$$
f_{0}\left(e_{1}\right):=e_{2}, f_{0}\left(e_{3}\right):=e_{4}, f_{0}\left(e_{1}+e_{3}\right):=e_{5}, f_{0}\left(e_{2}+e_{4}\right):=e_{6}
$$

[cf. (26) and (30)]. Furthermore

$$
f_{0}(0):=0 .
$$


No matter how we extend (31) to a function $f_{0}$ with domain $\mathbb{Z}_{2}^{6}$, this extension will certainly not be additive since

$$
f_{0}\left(e_{1}+e_{3}\right)=e_{5} \neq e_{2}+e_{4}=f_{0}\left(e_{1}\right)+f_{0}\left(e_{3}\right) .
$$

We write in the following $z \mapsto w$ for $f_{0}(z)=w\left(z, w \in \mathbb{Z}_{2}^{6}\right)$.

(31) with the assistance of (3) generates the following four 3 -cycles of $f_{0}$

$$
\begin{gathered}
e_{1} \mapsto e_{2} \mapsto e_{1}+e_{2} \mapsto e_{1}, \\
e_{3} \mapsto e_{4} \mapsto e_{3}+e_{4} \mapsto e_{3}, \\
e_{1}+e_{3} \mapsto e_{5} \mapsto e_{1}+e_{3}+e_{5} \mapsto e_{1}+e_{3}, \\
e_{2}+e_{4} \mapsto e_{6} \mapsto e_{2}+e_{4}+e_{6} \mapsto e_{2}+e_{4}
\end{gathered}
$$

2.2 ) For possibly finding the remaining seventeen 3 -cycles of $f_{0}$, our procedure is to put appropriate elements of $\mathbb{Z}_{2}^{6}$ into (1) in the places of $x$ and $y$ with the aim to determine a new pair $\left(w, f_{0}(w)\right)$, of course by the aid of 3 -cycles already computed; we then complete the 3 -cycle by means of (3). This program can in fact be realized, e.g., in the order in which the 3-cycles are listed in the following table, where the last element of every line is sent by $f_{0}$ to the first one.

\begin{tabular}{llll}
\hline$x$ & $y$ & resulting 3-cycle of $f_{0}$ & \\
\hline$e_{1}+e_{2}$ & $e_{3}$ & $e_{1}+e_{2}+e_{4} \mapsto e_{1}+e_{2}+e_{3}+e_{5} \mapsto e_{3}+e_{4}+e_{5}$ & $(38)$ \\
$e_{3}+e_{4}$ & $e_{1}$ & $e_{2}+e_{3}+e_{4} \mapsto e_{1}+e_{3}+e_{4}+e_{5} \mapsto e_{1}+e_{2}+e_{5}$ & $(39)$ \\
$e_{3}$ & $e_{2}$ & $e_{1}+e_{2}+e_{3} \mapsto e_{2}+e_{3}+e_{6} \mapsto e_{1}+e_{6}$ & $(41)$ \\
$e_{1}$ & $e_{4}$ & $e_{1}+e_{3}+e_{4} \mapsto e_{1}+e_{4}+e_{6} \mapsto e_{3}+e_{6}$ & $(42)$ \\
$e_{1}+e_{3}$ & $e_{3}$ & $e_{3}+e_{5} \mapsto e_{4}+e_{6} \mapsto e_{3}+e_{4}+e_{5}+e_{6}$ & $(43)$ \\
$e_{1}+e_{3}$ & $e_{1}$ & $e_{1}+e_{5} \mapsto e_{2}+e_{6} \mapsto e_{1}+e_{2}+e_{5}+e_{6}$ & $(45)$ \\
$e_{5}$ & $e_{1}$ & $e_{2}+e_{5} \mapsto e_{1}+e_{4}+e_{5}+e_{6} \mapsto e_{1}+e_{2}+e_{4}+e_{6}$ \\
$e_{5}$ & $e_{3}$ & $e_{4}+e_{5} \mapsto e_{2}+e_{3}+e_{5}+e_{6} \mapsto e_{2}+e_{3}+e_{4}+e_{6}$ \\
$e_{2}$ & $e_{6}$ & $e_{1}+e_{2}+e_{6} \mapsto e_{2}+e_{3}+e_{4}+e_{5} \mapsto e_{1}+e_{3}+e_{4}+e_{5}+e_{6}(46)$ \\
$e_{2}$ & $e_{2}+e_{3}+e_{6} e_{1}+e_{3}+e_{6} \mapsto e_{2}+e_{4}+e_{5}+e_{6} \mapsto e_{1}+e_{2}+e_{3}+e_{4}+e_{5}(47)$ \\
$e_{1}+e_{6}$ & $e_{1}$ & $e_{2}+e_{3} \mapsto e_{2}+e_{3}+e_{4}+e_{5}+e_{6} \mapsto e_{4}+e_{5}+e_{6}$ \\
$e_{2}+e_{6}$ & $e_{1}+e_{2}$ & $e_{5}+e_{6} \mapsto e_{1}+e_{2}+e_{3}+e_{4}+e_{5}+e_{6} \mapsto e_{1}+e_{2}+e_{3}+e_{4}(49)$ \\
$e_{1}$ & $e_{3}$ & $e_{1}+e_{4} \mapsto e_{1}+e_{2}+e_{4}+e_{5}+e_{6} \mapsto e_{2}+e_{5}+e_{6}$ \\
$e_{1}+e_{3}$ & $e_{2}+e_{4}$ & $e_{2}+e_{4}+e_{5} \mapsto e_{1}+e_{3}+e_{5}+e_{6} \mapsto e_{1}+e_{2}+e_{3}+e_{4}+e_{6}$ & $(51)$ \\
$e_{1}+e_{2}$ & $e_{4}+e_{5}$ & $e_{1}+e_{4}+e_{5} \mapsto e_{3}+e_{5}+e_{6} \mapsto e_{1}+e_{3}+e_{4}+e_{6}$ \\
$e_{2}+e_{5}+e_{6}$ & $e_{5}$ & $e_{1}+e_{2}+e_{3}+e_{6} \mapsto e_{2}+e_{3}+e_{5} \mapsto e_{1}+e_{5}+e_{6}$ \\
$e_{2}+e_{3}+e_{4}+e_{6}$ & $e_{1}$ & $e_{3}+e_{4}+e_{6} \mapsto e_{1}+e_{2}+e_{4}+e_{5} \mapsto e_{1}+e_{2}+e_{3}+e_{5}+e_{6}(54)$
\end{tabular}

Now $f_{0}: \mathbb{Z}_{2}^{6} \rightarrow \mathbb{Z}_{2}^{6}$ is explicitly defined by (32), (34),.., (54), is indeed bijective since the cycles were computed form a partition of $\mathbb{Z}_{2}^{6}$ and is not additive by (33). It remains to show that $f_{0} \in S_{0}\left(\mathbb{Z}_{2}^{6}\right)$ by inspecting the validity of $(1)$ for $f_{0}$ and all pairs $(x, y)$ of $\mathbb{Z}_{2}^{6} \times \mathbb{Z}_{2}^{6}$. It causes no principal problem to do this by hand, but it was done electronically. I cordially thank Hanspeter 
Bieri and Heinz Bruggesser, University of Bern, for their valuable assistance by writing and performing the corresponding computer program.

In total, $f_{0} \in S_{0}\left(\mathbb{Z}_{2}^{6}\right) \backslash \operatorname{End}\left(\mathbb{Z}_{2}^{6}\right)$, and Example 3.14 is established.

Corollary 3.15. If $2 G=\{0\}$ and $\operatorname{dim} G \geq \aleph_{0}$ or $\operatorname{dim} G \in 2 \mathbb{N}$ and $\geq 6$, then $S_{0}(G) \not \subset \operatorname{End}(G)$.

Proof. By Lemma 3.7, $S_{0}(G) \neq \emptyset$ in both cases. There are $\mathbb{Z}_{2}$-linear subspaces $M, N$ of $G$ with $G=M \oplus N$ and $\operatorname{dim} M=6$; therefore $M \cong \mathbb{Z}_{2}^{6}$. By Example 3.14 and Remark 1.1(c) $S_{0}(M) \not \subset \operatorname{End}(M)$; let $g_{0} \in S_{0}(M) \backslash$ $\operatorname{End}(M)$. Since $\operatorname{dim} N \geq \aleph_{0}$ or $\operatorname{dim} N \in 2 \mathbb{N}^{0}$, Lemma 3.7 guarantees $S_{0}(N) \neq$ $\emptyset$; choose $g_{1} \in S_{0}(N)$. By Lemma 2.3(a), $g: G \rightarrow G$ defined by $g\left(x_{1}, x_{2}\right):=$ $\left(g_{0}\left(x_{1}\right), g_{1}\left(x_{2}\right)\right)\left(\forall\left(x_{1}, x_{2}\right) \in M \times N=M \oplus N=G\right)$ belongs to $S_{0}(G)$. But $g_{0} \notin \operatorname{End}(M)$ implies $g \notin \operatorname{End}(G)$, so $S_{0}(G) \not \subset \operatorname{End}(G)$.

\section{References}

[1] Balcerowski, M.: On the functional equation $x+f(y+f(x))=y+f(x+f(y))$. Aequationes Math. 75, 297-303 (2008)

[2] Brillouët-Belluot. N., Problem 15. Report of Meeting. The Thirty-eighth International Symposium on Functional Equations (Noszvaj, 2000). Aequationes Math. 61, 304 (2001)

[3] Jarczyk, J., Jarczyk, W.: On a problem of N Brillouët-Belluot. Aequationes Math. 72, 198-200 (2006)

[4] McShane, N.: On the periodicity of homeomorphisms of the real line. Am. Math. Mon. 68, 562-563 (1961)

[5] Rätz, J.: On the functional equation $x+f(y+f(x))=y+f(x+f(y))$. Report of Meeting. The Forty-eighth International Symposium on Functional Equations (Batz-sur-Mer, 2010). Aequationes Math 81, 300 (2011)

[6] Suzuki, M.: Group theory I Grundlehren der mathematischen Wissenschaften 247. Springer, Berlin (1982)

Jürg Rätz

Mathematisches Institut der Universität Bern

Sidlerstrasse 5, 3012 Bern, Switzerland

e-mail: math@math.unibe.ch

Received: January 18, 2013 